\title{
Técnicas histórico-etnográficas en la reconstrucción y caracterización de tsunamis: el ejemplo del gran tsunami del 22 de junio de 1932, en las costas del Pacífico mexicano ${ }^{1}$
}

\author{
Néstor Corona Morales² y María Teresa Ramírez-Herrera ${ }^{3}$
}

\begin{abstract}
RESUMEN
La magnitud de los desastres ocasionados por tsunamis en la última década ha motivado a ampliar el conocimiento sobre su dinámica y comportamiento. Este estudio propone la aplicación de técnicas de análisis multidisciplinarias, incluyendo las histórico-etnográficas en la reconstrucción y caracterización de los tsunamis sin un registro instrumental. Se usa el ejemplo del tsunami del 22 de junio de 1932, el segundo más destructivo registrado en las costas del Pacífico mexicano. El origen del tsunami es incierto y se plantean dos hipótesis: 1) sísmico, y 2) por un deslizamiento submarino. Archivos históricos, entrevistas y mapeo en Sistemas de Información Geográfica permitieron identificar componentes clave de la dinámica del tsunami: tiempo de arribo, directividad, superficie afectada y alturas máximas de ola en la costa. Se aplicaron modelos numéricos (GEOWAVE y FUNWAVE) basados en datos históricos, usando dos posibles mecanismos de generación. Los resultados demuestran que un deslizamiento submarino explica mejor la causa del tsunami de 1932.
\end{abstract}

Palabras clave: Datos históricos, enfoque etnográfico, tsunamis, tsunami-deslizamientos, modelación de tsunamis.

\begin{abstract}
The magnitude of damage caused by tsunamis in the past decade has encouraged scientists to expand our knowledge about tsunami dynamics and behavior. This study applies a multidisciplinary analysis, including historical and ethnographic techniques, in the reconstruction and characterization of tsunamis with no instrumental record. The example of the 22 June 1932 tsunami, the second most destructive recorded in the Pacific Coast of Mexico is used. The cause of this tsunami is uncertain. Two hypotheses are proposed: 1) seismic slip, and 2) a submarine landslide. Historical archives, interviews with local witnesses, and GIS mapping, aided in identifying key components of the tsunami dynamics: arrival time, directivity, affected area, and maximum wave heights at the coast. Based on historical data, we applied numerical models (GEOWAVE and FUNWAVE codes) to the two possible mechanisms of tsunami generation. The results show that a submarine landslide explains best the cause of the 1932 tsunami.
\end{abstract}

Key words: Historical data, ethnographic approach, tsunami, landslide tsunami, tsunami modeling.

\footnotetext{
1 N. Corona agradece al CONACYT por proporcionar la beca para realizar los estudios de Doctorado. M.T. Ramírez-Herrera agradece a PAPPIT UNAM por proporcionar los fondos de investigación mediante el apoyo IN123609 y a SEP-CONACYT por el apoyo $N^{\circ}$ 129456. Se agradece a P. Urquijo, por su apoyo en el tratamiento y búsqueda de fuentes hemerográficas. Artículo recibido el 15 de agosto de 2012 aceptado el 20 de septiembre de 2012 y corregido el 29 de septiembre de 2012
}

2 Centro de Estudios en Geografía Humana, El Colegio de Michoacán y Centro de Investigaciones en Geografía Ambiental, Universidad Nacional Autónoma de México (México).

E-mail: ncorona@pmip.unam.mx

3 Centro de Investigaciones en Geografía Ambiental, Universidad Nacional Autónoma de México y Laboratorio Universitario de Geofísica Ambiental, Universidad Nacional Autónoma de México (México). E-mail:mtramirez@ciga.unam.mx 
En la última década, los desastres y daños causados por los tsunamis han sido de dimensiones gigantescas e incomparables a las de otros fenómenos naturales, tan solo el tsunami del 11 de marzo de 2011, causó el $64.5 \%$ de las muertes asociadas a desastres naturales (Guha-Sapir et al., 2012). Uno de los principales retos para mitigar los efectos de los tsunamis implica en gran medida el conocimiento de la probabilidad de ocurrencia y la magnitud del mismo. Al ser eventos de baja frecuenta, gran potencial destructivo (Birkmann \& Fernando, 2008), presentan un reto, ya que se trata de un campo de investigación en constante desarrollo, basta señalar el cambio de paradigma del potencial sísmico y tsunamigénico en zonas de subducción que antecedía al tsunami del 2004 en el océano Índico (Stein, 2006).

Los modelos de valoración de peligro por tsunamis se basan principalmente en análisis de datos de sismos con potencial tsunamigénico. Una forma de estimar el periodo de retorno de los tsunamis se deriva del análisis de las tasas de convergencia en zonas de subducción, para determinar la cantidad de energía que se puede acumular en la interfaz de los bloques y derivar la magnitud del sismo resultante de dicho esfuerzo, la deformación del fondo oceánico y por tanto la probabilidad de que un tsunami se presente (Løvholt et al., 2012). Otro enfoque consiste en el análisis estadístico de datos sísmicos, el principal inconveniente es la escasa cobertura histórica de los registros sísmicos. Un tercer enfoque consiste en determinar el peligro de tsunamis a partir de las estadísticas de run-up provenientes de catálogos (Løvholt et al., 2012). Este grupo de modelos se pueden aplicar cuando se cuenta con mediciones instrumentales, sin embargo, las prácticas de recolección de información instrumental se viene realizando solamente desde la segunda mitad del siglo XVII (Fliri, 1998; Barriendos et al., 2003; Llasat et al., 2005). Los sismos y tsunamis cuyos periodos de gestación superan la cobertura de los datos instrumentales, difícilmente pueden ser analizados con estos enfoques, i.e., el predecesor del megatsunami de 2004 en el océano Índico ocurrió entre 550-700 años (Jankaew et al., 2008).

Por otro lado, se ha recurrido al empleo de modelos determinísticos, en los cuales, si bien no es posible determinar periodos de retorno, es posible identificar las alturas máximas de tsunamis extremos mediante el análisis de registros observacionales históricos (e.g. Kulikov et al., 2005 y Usha et al., 2012). En general, los datos históricos pueden ser más directos, claros, y en la mayoría de los casos más reveladores que datos generados con técnicas que miden parcialidades de los fenómenos (Wang \& Zhang, 1988). Aun tratándose de meras descripciones, al ser validados pueden generar datos importantes en el entendimiento de fenómenos naturales (Barnikel, 2004; Giannecchini \& D'Amato Avanzi, 2012).

\section{El tsunami del 22 de junio de 1932, su contexto sismo- tectónico y tsunamigénico}

La costa del Pacífico mexicano se ubica en la zona limítrofe y de convergencia de las placas Norteamericana (NAP), de Cocos (CP) y de Rivera (RP); definida por la Trinchera Mesoamericana (MAT), en donde subducen las placas de Rivera y Cocos bajo la NAP (Singh et al., 1981; Nixon, 1982; Eissler \& McNally, 1984; Luhr et al., 1985; Singh et al., 1985). En la zona de subducción de las costas mexicanas se han presentado 70 tsunamis entre 1732-1993 (Sánchez y Farreras, 1993; National Geophysical Data Center, 2011; Novosibirsk Tsunami Laboratory, 2011), de los cuales probablemente 27 hayan tenido alguna manifestación en Cuyutlán, siendo el del 22 de junio de 1932 el de mayor magnitud (Corona \& Ramírez-Herrera, 2012b).

El tsunami del 22 de junio de 1932, se presentó en un contexto sísmico muy activo (Ordóñez, 1933). La cadena sismos y tsunamis afectaron una gran extensión de las costas de Nayarit, Jalisco y Colima (Figura $N^{\circ} 1$ ). El sismo principal se presentó el 3 de junio de 1932, con una magnitud de 8.1 Ms (Singh et al., 1981; Wyss et al., 1984, Singh et al., 1985) provocando un tsunami que afectó a la bahía de Manzanillo, con alturas máximas de 3 m (Sánchez y Farreras, 1993) y localidades situadas al NW de esta, particularmente Mismaloya, localizada a $\sim 160 \mathrm{~km}$ de la bahía de Manzanillo, en la que se registró una altura máxima de $10 \mathrm{~m}$ (Valdivia-Ornelas et al., 2011). El segundo sismo se presentó el 
18 de junio (7.8 Ms), afectando a la bahía de Manzanillo con alturas máximas de $1 \mathrm{~m}$; el tercero corresponde al sismo del 22 de junio, de $M s=6.9$, el cual produjo un tsunami que generó olas de hasta $10 \mathrm{~m}$ en la localidad de Cuyutlán, en la cual se reportó la muerte de 50 personas, cerca de 1.500 afectados (El Informador, 1932b; El Universal Gráfico, 1932a; Excelsior, 1932d; Ordóñez, 1933) y la destrucción total de varios asentamientos costeros (Chávez, 1932; Cumming, 1933).

El mecanismo de generación del tsunami del 22 de junio aún no ha sido definido (Singh et al., 1985), se explica parcialmente mediante dos hipótesis: 1) por sismo (Sánchez y Farreras, 1993; Singh et al., 1998; National Geophysical Data Center, 2011; Okal \& Borrero, 2011), y 2) por deslizamiento submarino (El Informador, 1932a; Excelsior, 1932c; Cumming, 1933). Parte de las incógnitas surgen por el hecho de que a pesar de que el sismo del 22 de junio presentó valores de magnitud y momento sísmico pequeño (Okal
\& Borrero, 2011), ocasionó alturas máximas de ola de 10-12 m (Farreras \& Sánchez, 1991).

Okal \& Borrero (2011), basados en el trabajo de Fukao (1979), proponen que el tsunami fue generado por un mecanismo "sismotsunamigénico" que se produjo en una falla adyacente a la falla principal (Splay fault), capaz de generar tsunamis de mayor magnitud que la esperada (Fukao, 1979). Sin embargo, en su modelo de mejor ajuste, reportan alturas máximas de ola de hasta $7 \mathrm{~m}$ en la bahía de Manzanillo y en Cuyutlán, valores que no se ajustan a los datos reportados. En Manzanillo no hay reportes de daños y en Cuyutlán la altura de ola fue superior a $10 \mathrm{~m}$ (El Universal Gráfico, 1932b; Excelsior, 1932b; Soloviev \& Go, 1975; Salazar, 1985; Farreras \& Sánchez, 1991; Sánchez y Farreras, 1993). El modelo propuesto presenta, además, una distribución homogénea, es decir, alturas de 7 metros a lo largo de toda la costa de Colima.

Figura $\mathrm{N}^{\circ} 1$

Contexto sismo-tectónico y cadena de tsunamis de mayores dimensiones en el mes de junio de 1932. Símbolos: a) y b) Epicentros de los sismos del 3 y 18 de junio, respectivamente (Cumming, 1933), c) Epicentro del sismo del 22 de junio (Servicio Sismológico Nacional, 2011). B) Registro histórico de tsunamis (National Geophysical Data Center, 2011).

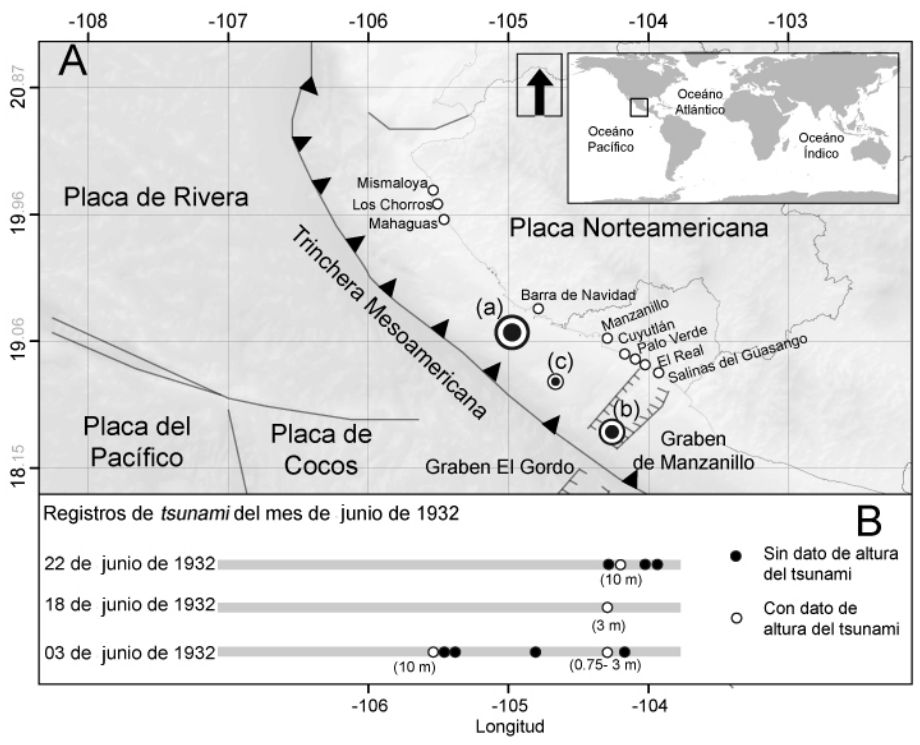

Fuente: Elaboración propia. 
Por otro lado, los modelos que apoyen la tesis de un deslizamiento submarino como causa del tsunami, carecen hasta ahora de argumentos sólidos (El Informador, 1932a; Excelsior, 1932c) o están basados en observaciones directas en campo (Cumming, 1933).

En la presente investigación se efectuó la caracterización del tsunami a partir de información histórica y testimonios de sobrevivientes. Los resultados de la caracterización histórico-etnográfica fueron comparados con los patrones de comportamiento de tsunamis generados por causas sísmicas y por deslizamientos. A su vez, dichos patrones fueron generados a partir de la modelación numérica de generación y propagación de tsunamis por ambas condiciones.

\section{Métodos}

La estrategia de investigación consistió en: 1) caracterización del tsunami a partir de de datos históricos -tiempo de arribo, dirección de la propagación, área afectada y amplitud máxima del tsunami en la costa- obtenidos mediante el uso de técnicas históricas, etnográficas y geográficas; 2) modelado numérico de los tsunamis por causas sísmicas y por deslizamiento submarino, y 3) comparación de datos compilados histórico-etnográficos y de datos modelados.

Las técnicas históricas-etnográficas consintieron en el análisis de contenido de: archivos históricos, material hemerográfico, reportes técnicos, artículos científicos y catálogos, así como la aplicación de entrevistas a testigos presenciales o a sus descendientes. Las técnicas geográficas consistieron en representar los atributos del tsunami, cualitativos como cuantitativos, sobre mapas históricos.

La modelación de los tsunamis tuvo como objetivo obtener los parámetros de alturas máximas de tsunami en la costa, dirección de propagación, tiempos de arribo e identificación de la extensión de la costa que fue afectada. Los modelos fueron generados con los códigos de modelación GEOVAWE y FUNWAVE. Los parámetros de la modelación por causas sísmicas (MGTS) (Cuadro $N^{\circ} 1$ ) y por deslizamientos (Cuadro $\mathrm{N}^{\circ} 2$ ) fueron tomados de Corona \& Ramírez-Herrera (2012a). Los datos batimétricos fueron tomados de la colección del Global Multi-Resolution Topography de Ryan et al. (2009) obtenida mediante el software GeoMapApp 3.0.1, y generalizada a pixeles de $\sim 200 \times 200 \mathrm{~m}$.

Cuadro $\mathrm{N}^{\circ} 1$

Parámetros sísmicos de la modelación de las condiciones iniciales del tsunami

\begin{tabular}{|l|r|}
\hline Parámetro & Valor \\
\hline Longitud del centroide de la falla $\left(^{\circ}\right)$ & -104.680 \\
\hline Latitud del centroide de la falla $\left(^{\circ}\right)$ & 18.739 \\
\hline Strike $\left(^{\circ}\right)$ & 310 \\
\hline Dip $\left(^{\circ}\right)$ & 14 \\
\hline Rake $\left(^{\circ}\right)$ & 90 \\
\hline Magnitud del sismo $(\mathrm{Ms})$ & 6.9 \\
\hline Profundidad $(\mathrm{m})$ & 30.000 \\
\hline Longitud de la falla $(\mathrm{m})$ & 80.000 \\
\hline Ancho de la falla $(\mathrm{m})$ & 40.000 \\
\hline Modulo de corte $(\mathrm{N})$ & $4 \mathrm{E}+10$ \\
\hline Profundidad del piso oceánico & 860 \\
\hline
\end{tabular}

Fuente: Ajustada a partir de Corona y Ramírez-Herrera (2012a). 

DE TSUNAMIS: EL EJEMPLO DEL GRAN TSUNAMI DEL 22 DE JUNIO DE 1932, EN LAS COSTAS DEL PACÍFICO MEXICANO

Cuadro $\mathrm{N}^{\circ} 2$

Parámetros de la modelación por deslizamiento submarino

\begin{tabular}{|l|r|}
\hline Parámetro & Valores \\
\hline$X=$ Longitud & -104.0376 \\
\hline$Y=$ Latitud & 18.7586 \\
\hline$y=$ Densidad específica & 1.906 \\
\hline$b(\mathrm{~km})=$ Longitud del cuerpo del deslizamiento & 3.5 \\
\hline$T(\mathrm{~m})=$ Espesor máximo del deslizamiento & 450 \\
\hline$\omega(\mathrm{km})=$ Ancho máximo del cuerpo del deslizamiento & 3.0 \\
\hline$d(\mathrm{~m})=$ Profundidad promedio del deslizamiento & 460 \\
\hline$\theta\left({ }^{\circ}\right)=$ Pendiente promedio & 6.0 \\
\hline
\end{tabular}

Fuente: Ajustada a partir de Corona y Ramírez-Herrera (2012a).

La modelación del tsunami generado por deslizamiento (MGTD) se realizó usando como insumos los parámetros de Corona y Ramírez-Herrera (2012a), plantean que el tsunami se generó por la combinación de dos eventos consecutivos: 1) el sismo de $6.9 \mathrm{Ms}$ (Singh et al., 1985), tomando los mismos parámetros del modelo sísmico, y 2) un deslizamiento submarino de tipo rotacional (slump) que fue desencadenado 10 segundos después de la generación de sismo.

\section{Caracterización del tsunami en base a datos histórico- etnográficos}

La revisión hemerográfica estuvo comprendida por 46 notas periodísticas de diarios de circulación nacional, publicadas entre el 23 y el 30 de junio de 1932, de las cuales destacaron 13 publicaciones que enlistan elementos referentes a los datos requeridos para la caracterización del tsunami (Cuadro $N^{\circ} 3$ ). De igual forma se consultaron 206 notas de prensa internacional, estadounidense, de las cuales destacó solo una nota (Chávez, 1932), por tratarse de una transcripción literal del diario Ecos de la Costa, periódico de circulación local, misma que se perdió en un incendio del archivo del periódico. La revisión documental se centró en: el informe técnico de Cumming (1933), El catálogo tsunamis de Soloviev y Go (1975), El catálogo de tsunamis de Sánchez y Farreras (1993) y la descripción de Salazar (1989). Las entrevistas fueron aplicadas a: un testigo ocular, doña Pachita Gaspar (92 años), a hijos de testigos oculares: el Sr. Ramón Cortez (70 años), al Sr. Vidal Mojarro Vázquez (76 años), Sra. María de Jesús Morales (50 años), Ignacio de la Madrid (78 años) y esposa (76 años), al Sr. José Solórzano (52 años) y al cronista de municipio de Armería, Lic. Miguel Chávez Michel (62 años). Los datos fueron clasificados de acuerdo a diez categorías de información cuya lectura permitió identificar o inferir las características del tsunami, extensión afectada, altura del tsunami, directividad y tiempo de arribo (Ver Cuadro $\mathrm{N}^{\circ} 3$ ).

Los resultados indican que a las 7:00 (UTC-6) del 22 de junio de 1932 se produjo un sismo de $\mathrm{Ms}=6.9$ con epicentro a $\sim 86$ $\mathrm{km}$ de la bahía de Manzanillo (Soloviev \& Go, 1975; Singh et al., 1981; Cruz y Wyss, 1983; Singh et al., 1985; Farreras \& Sánchez, 1991; Sánchez \& Farreras, 1993; Servicio Sismológico Nacional, 2011). Cinco minutos después (7:05 UTC-6), un tsunami con alturas de olas de más de $10 \mathrm{~m}$ golpeó la costa del estado de Colima, desde la bahía de Manzanillo hasta por lo menos las Salinas del Guasango (Sánchez y Farreras, 1993), penetrando en algunos sectores hasta $2 \mathrm{~km}$ en el continente (El Universal Gráfico, 1932b, 1932c; Excelsior, 1932b; Soloviev \& Go, 1975; Salazar, 1985; Sánchez y Farreras, 1993). 
Cuadro $\mathrm{N}^{\circ} 3$

Relación de fuentes de información consultadas

\begin{tabular}{|c|c|c|c|c|}
\hline Tipo de fuente & \multicolumn{2}{|l|}{ Referencia } & Clave & Categoría del dato \\
\hline \multirow{13}{*}{ Hemergráfica } & \multirow{2}{*}{ El Informador } & $(1932 a)$ & (1) & $d, j$ \\
\hline & & (1932b) & (2) & $b, d$ \\
\hline & El Nacional (1932) & & (3) & i \\
\hline & \multirow{3}{*}{ El Universal Gráfico (1932) } & $(1932 a)$ & (4) & $b, d, e, f$ \\
\hline & & (1932b) & (5) & $b, g, i$ \\
\hline & & (1932c) & (6) & $\mathrm{h}$ \\
\hline & \multirow{5}{*}{ Excelsior } & $(1932 a)$ & (7) & $d, f, i$ \\
\hline & & (1932b) & (8) & $a, b, d, h, i$ \\
\hline & & (1932c) & (9) & i \\
\hline & & $(1932 d)$ & (10) & $d, j$ \\
\hline & & $(1932 e)$ & (11) & d, g \\
\hline & Salazar (1985). & & (12) & $a, b, d, e, f$ \\
\hline & El Nuevo Mexicano (1932). & & (13) & a d, e, h \\
\hline Libros & Salazar (1989). & & (14) & $a, b, d, e, f, h$ \\
\hline Reportes técnicos & Cumming (1933). & & (15) & $a, b, c, d, e, f, g, h, i$ \\
\hline \multirow{2}{*}{ Entrevistas } & Sobrevivientes & & (16) & $a, b, d, e, f, k$ \\
\hline & Hijos de sobrevivientes & & $(17)$ & $a, b, d, e, f, k$ \\
\hline \multirow{4}{*}{ Catálogos } & Soloviev \& Go (1984). & & (18) & $a, b, d, e, f$ \\
\hline & Sánchez y Farreras (1993). & & $(19)$ & $a, b, d, e, f, g$ \\
\hline & NGDC (2011). & & $(20)$ & $a, b, e$ \\
\hline & HTDB/WLD (2011). & & $(21)$ & $a, b, e$ \\
\hline \multirow{5}{*}{$\begin{array}{l}\text { Artículos cientí- } \\
\text { ficos }\end{array}$} & Ordóñez (1933). & & $(22)$ & $a, b, e$ \\
\hline & Flores (1934). & & $(23)$ & $a, b, e$ \\
\hline & Farreras \& Sánchez (1991). & & $(24)$ & $a, b, d, e, f, g$ \\
\hline & Okal \& Borrero (2011). & & $(25)$ & $a, b, i$ \\
\hline & Corona \& Ramírez-Herrera (2012). & & $(26)$ & $a, b, c, d, e, f, g, h, i, j$ \\
\hline
\end{tabular}

Notas: Claves de los tipos de dato: a) Altura del tsunami, b) nombres de localidades afectadas, c) dirección del tsunami, d) daños en sitios específicos, e) hora de arribo del tsunami, f) datos puntuales de inundación, g) extensión afectada, h) fotografías, i) origen del tsunami, j) acciones de contingencia.

Fuente: Elaboración propia.

Las localidades que reportaron el tsunami fueron Manzanillo, Cuyutlán, Palo Verde, Las Boquillas, El Real y Tecuanillo (Salinas de Guasango) y Boca de Apiza (ver Cuadro Nº 4). Cuyutlán fue la localidad que más daños sufrió, además de ser la localidad que aparece en todas las fuentes de información. Man- zanillo, aunque cuenta con el registro del $t s u$ nami, no tiene con datos de la altura de este. La localidad de Palo Verde, situada a $3 \mathrm{~km}$ al SE de Cuyutlán, fue totalmente devastada. En la localidad de Las Boquillas, el mar penetró hasta unirse con el estero. La localidad de El Real también fue arrastrada por el tsunami. En 
cuanto a Tecuanillo, que se localizaba prácticamente en los alrededores de las Salinas del Guasango, sufrió daños similares a los de Palo Verde. Finalmente, los entrevistados señalan que el tsunami alcanzó a la localidad de Boca de Apiza, pero que en este punto no se reportaron daños. A partir de la distribución geográfica de las localidades afectadas, algunos reportes de prensa señalan que la longitud de afectación fue de $25 \mathrm{~km}$, desde Cuyutlán hasta la localidad de El Tecuanillo (Salinas del Guasango).

El Cuadro $N^{\circ} 4$ presenta la relación de las localidades que reportaron el tsunami, algunos detalles de su comportamiento en ese punto y la relación de fuentes de información en las que se hace referencia a cada una.

En cuanto a la directividad del tsunami, solo el reporte técnico de Cumming (1933) hace referencia a este atributo del tsunami. Menciona que por la dirección que tomaron los árboles y postes arrasados frente a la playa de Palo Verde y El Guasango, parece que el centro de propagación del tsunami está situado a unos $10 \mathrm{~km}$ al S-SW de la Boca de Pascuales (c.a. $3 \mathrm{~km}$ al SE de Las Boquillas).

Las características del tsunami fueron mapeadas sobre cartografía antigua que contiene a todas las localidades enunciadas en las descripciones (Figura $\mathrm{N}^{\circ} 2$ 2). El mapa captura la visión completa del tsunami, considerando los aspectos de localización y directividad observada del tsunami de acuerdo a las notas técnicas de Cumming (1933), quien sitúa la fuente tsunamigénica al S-SW de la desembocadura del río Armería (Figura $N^{\circ} 2$ ).

\section{Modelación del tsunami originado por el sismo (MGTS) de $M s=6.9$ \\ del 22 de junio de 1932}

\section{Altura máxima del tsunami en la costa y superficie afectada}

La Figura $N^{\circ} 3$ muestra la distribución de las alturas máximas del tsunami en la línea de Costa causadas por el tsunami del 22 de

Cuadro $\mathrm{N}^{\circ} 4$

Listado de localidades afectadas según fuente de información

\begin{tabular}{|c|c|c|}
\hline Localidades afectadas & Descripción & Fuente* \\
\hline Manzanillo & $\begin{array}{l}\text { Sin daños registrados, solo repor- } \\
\text { ta el tsunami }\end{array}$ & (4), (5), (7), (18), (19), (21), (24) y (25). \\
\hline Cuyutlán & $\begin{array}{l}\text { Totalmente destruido con olas de } \\
\text { más de } 10 \text { metros }\end{array}$ & $\begin{array}{l}(1),(2),(3),(4),(5),(6),(7),(8),(9),(10),(11), \\
(12),(13),(14),(15),(16),(17),(18),(19), \\
(20),(21),(22),(23),(24),(25),(26),(27) y \\
(28) .\end{array}$ \\
\hline Palo Verde & Totalmente destruido & $\begin{array}{l}(1),(2),(3),(4),(5),(6),(7),(8),(9),(10),(11), \\
(12),(13),(14),(15),(16),(17),(18),(19), \\
(20),(21),(22),(23),(24),(25) \text { y }(26) .\end{array}$ \\
\hline Las Boquillas & Se unió el mar con el estero & $(12),(13),(16)$ y (17). \\
\hline El Real & $\begin{array}{l}\text { Registro sin dato de altura de } t s u- \\
\text { nami, pero con detalle de daños }\end{array}$ & $\begin{array}{l}(1),(2),(3),(4),(5),(6),(7),(8),(9),(10),(11), \\
(12),(13),(14),(15),(16),(17),(18),(19), \\
(20),(21),(22),(23),(24),(25) \text { y }(26) .\end{array}$ \\
\hline $\begin{array}{l}\text { Tecuanillo (Salinas de } \\
\text { Guasango) }\end{array}$ & Daños similares a Palo Verde & (8), (15), (18), (19), (20) y (21). \\
\hline Boca de Apiza & Sin reportes de daños & (16) y (17). \\
\hline
\end{tabular}

* Las claves numéricas de esta columna corresponden a la columna 4 (Clave) del Cuadro $\mathrm{N}^{\circ} 3$.

Fuente: Elaboración propia. 
Figura $\mathrm{N}^{\circ} 2$

Mapeo de las descripciones del tsunami del 22 de junio de 1932

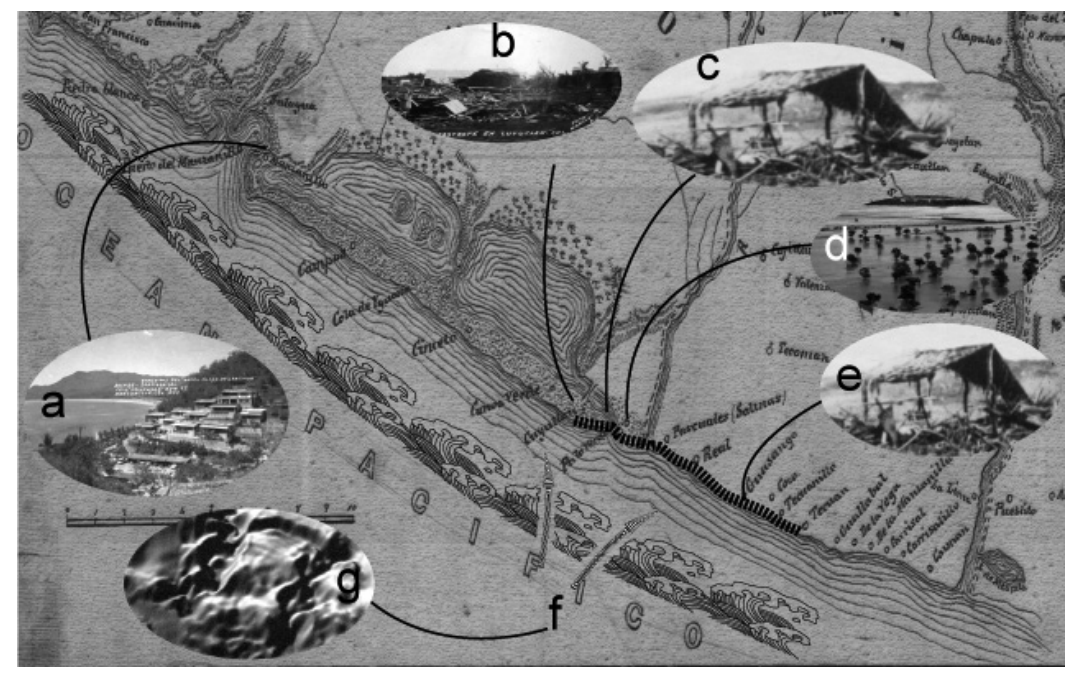

Notas: a) Bahía de Manzanillo, b) Cuyutlán, c) Palo Verde, d) Las Boquilas, e) Tecuanillo (Salinas del Guasango), f) probable zona de generación del tsunami, g) gráfico alusivo al deslizamiento. Símbolos: Línea discontinua en la línea de costa, longitud de mayor afectación.

Fuente: Elaboración propia en base al plano histórico de Matute (1865).

junio originado por causa sísmica. Los valores máximos de altura del tsunami resultantes de esta modelación oscilan entre los 0.08 y 2.2 $\mathrm{m}$. El valor máximo de $2.2 \mathrm{~m}$ se presenta en la sección noroeste del interior de la bahía de Manzanillo (Figura $\mathrm{N}^{\circ} 3$ ). En el resto de la costa, presenta valores de altura máxima del tsunami inferiores a $1.2 \mathrm{~m}$, y mantiene un promedio de $0.6 \mathrm{~m}$, valores que se extienden a lo largo de la costa en una longitud de cerca de $75 \mathrm{~km}$. Específicamente, en la localidad de Cuyutlán los valores resultantes son de entre 0.3 y $0.55 \mathrm{~m}$.

\section{Tiempo de arribo y directividad}

El tiempo estimado de arribo (TEA) a Cuyutlán resultó entre 12 y 12.5 min. Para la bahía de Manzanillo, entre 9 min., en la boca de la bahía, y $18 \mathrm{~min}$ en la zona del puerto y playa. Para lo localidad de Palo Verde es de 12 a $12.5 \mathrm{~min}$. En la localidad de Las BoquiIlas (Paraíso) los tiempos son de 11.5 a 11.7 min. Para la localidad de El Real de entre 14.5 y 15.6 min, y en la localidad de Salinas del Guasango (Tecuanillo) de $15 \mathrm{~min}$. Final- mente, el extremo SE de la costa afectada, en la localidad de Boca de Apiza, los tiempos son del orden de entre 15 y 16 min (ver Figura $\mathrm{N}^{\circ} 4$ ).

La dirección de los flujos de propagación del tsunami se extiende de forma perpendicular al eje de ruptura del sismo, en dirección WSW-ENW, y conserva este patrón desde la localidad de Manzanillo hasta la localidad de Salinas del Guasango (Tecuanillo).

\section{Modelación del tsunami originado por un deslizamiento submarino (MGTD) el 22 de junio de 1932}

Distribución de las alturas máximas del
tsunami, longitud de costa afectada

El modelo presenta una concentración de los valores máximos de altura del tsunami en el segmento de costa comprendido entre la localidad de Cuyutlán y rumbo al SE, hasta 
Figura $N^{\circ}$ 3. Máxima deformación de la superficie oceánica a causa del sismo

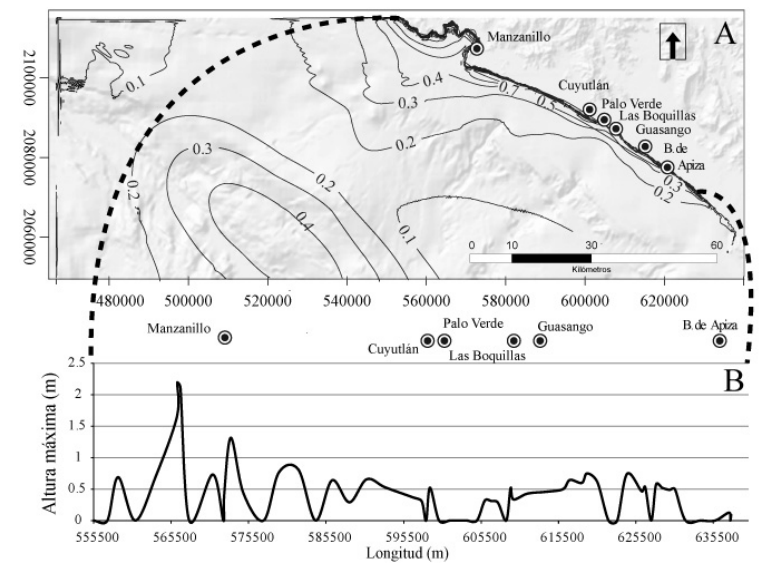

A) Deformación máxima de la superficie oceánica. Las líneas negras representan isolíneas a intervalos de 0.1 metro. B) Perfil de la altura máxima del tsunami en la costa.

Fuente: Elaboración propia.

Figura $N^{\circ} 4$

Tiempos de arribo del tsunami y directividad del modelo de generación por sismo. Símbolos: las flechas indican la dirección del flujo; líneas negras, las isócronas del TEA con intervalos de 0.5 minutos

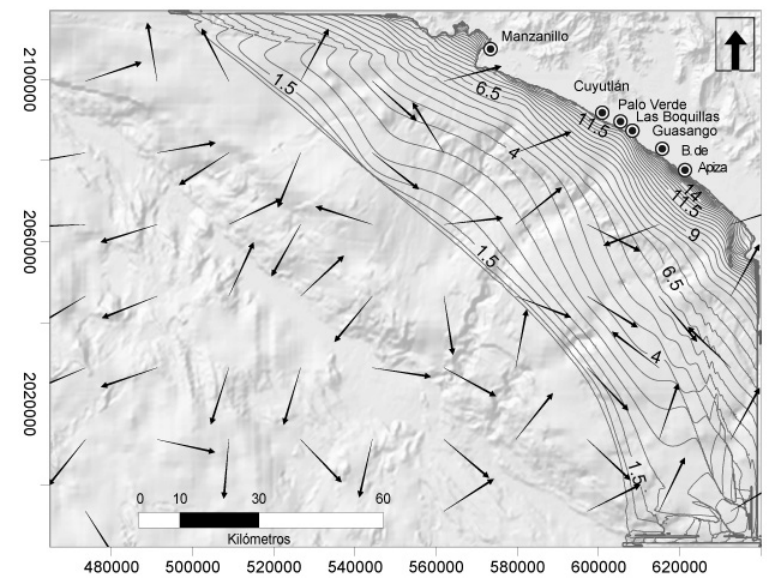

Fuente: Elaboración propia.

la localidad de El Tecuanillo (una sección de $\sim 26 \mathrm{~km}$ ), el resto de la línea de costa presenta valores de altura máxima de entre 0.5 y $2 \mathrm{~m}$. Los valores que se presentan al interior de la bahía de Manzanillo están entre 0.9 y 2.3 m. (Figura $\mathrm{N}^{\circ} 5$ ). Los valores resultantes en la localidad de Cuyutlán son de entre 8.4 - 11.6 m
(Figura $\mathrm{N}^{\circ}$ 5). En la localidad de Palo Verde resultaron de 7.9 y 8 m (Figura $\mathrm{N}^{\circ} 5$ ). En la localidad de Las Boquillas (actualmente la localidad de El Paraíso) de $8.7 \mathrm{~m}$. En la localidad de El Real, el valor es de $12.1 \mathrm{~m}$. En la localidad de Salinas del Guasango (actual poblado de Tecuanillo), los valores son de entre 4.3 y $6.4 \mathrm{~m}$. Por último, para la localidad 
de Boca de Apiza, son de entre 1.7 y $1.9 \mathrm{~m}$ (Figura $\mathrm{N}^{\circ}$ 5).

\section{Tiempo de arribo y directividad}

El tiempo estimado de arribo (TEA) a Cuyutlán es de 8.5 minutos. Para la bahía de Manzanillo, los valores de TEA son de entre 9 (en la boca de la Bahía) a 14 min. en la zona del puerto y playa. Para lo localidad de Palo Verde es de de $7 \mathrm{~min}$. En la localidad de Las Boquillas (Paraíso) el tiempo resulto en 6 min. Para la localidad de Salinas del Guasango (Tecuanillo) es de $10 \mathrm{~min}$. Finalmente, en el extremo sureste, en la localidad de Boca de Apiza, los tiempos son del orden de entre 15 y 16 min (ver Figura $N^{\circ} 6$ ).

La dirección de los flujos de propagación del tsunami se extiende de forma perpendicular al eje de la falla del deslizamiento y con un fuerte componente SSE-NNW (Figura $N^{\circ}$ 6). El ángulo de ataque en la localidad de Cuyutlán y Palo Verde tiene un ligero compo- nente SSE. En las localidades de Las Boquillas (Paraíso), El Real y Salinas del Guasango la dirección predominante es SW-NE. En la localidad del Tecuanillo la dirección del flujo es perpendicular a la línea de costa. Para la localidad de Boca de Apiza, los flujos se disipan drásticamente y corren en sentido NW$\mathrm{SE}$, casi paralelo a la línea de costa (Figura $\left.N^{\circ} 6\right)$.

\section{Discusión, comparación de los datos histórico-etnográficos versus los modelos numéricos}

Distribución de las alturas del tsunami y extensión afectada

Se encontró que los registros de las alturas máximas del tsunami son escasos, de las pocas fuentes que reportan este dato se puede mencionar el Catálogo de Tsunamis Históricos del National Geophysical Data Center (2011), el cual tiene el registro de cin-

Figura $\mathrm{N}^{\circ} 5$

Máxima deformación de la superficie oceánica a causa del deslizamiento submarino. Símbolos: A) Deformación máxima de la superficie oceánica. Las líneas negras representan isolíneas a intervalos de $0.2 \mathrm{~m}$ en las zonas más con menor deformación y de $1 \mathrm{~m}$ en las zonas más cercanas a la zona de generación del tsunami. B) Perfil de la altura máxima del tsunami en la costa.

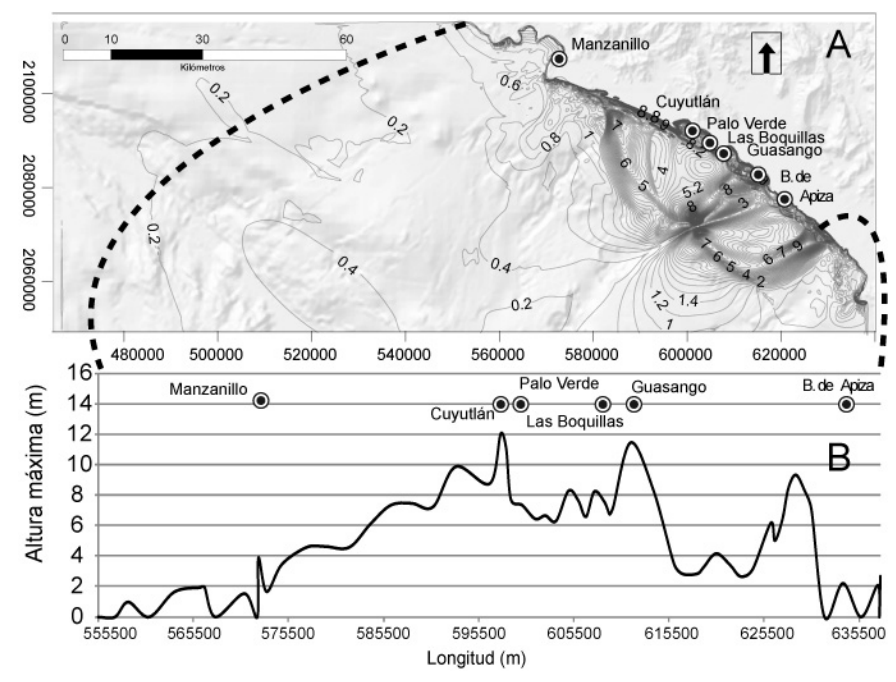

Fuente: Elaboración propia. 
Figura $\mathrm{N}^{\circ} 6$

Tiempos de arribo del tsunami y directividad del modelo de generación por deslizamiento submarino. Símbolos: Las flechas indican la dirección del flujo. Líneas negras, las isócronas del

TEA con intervalos de 0.5 minutos

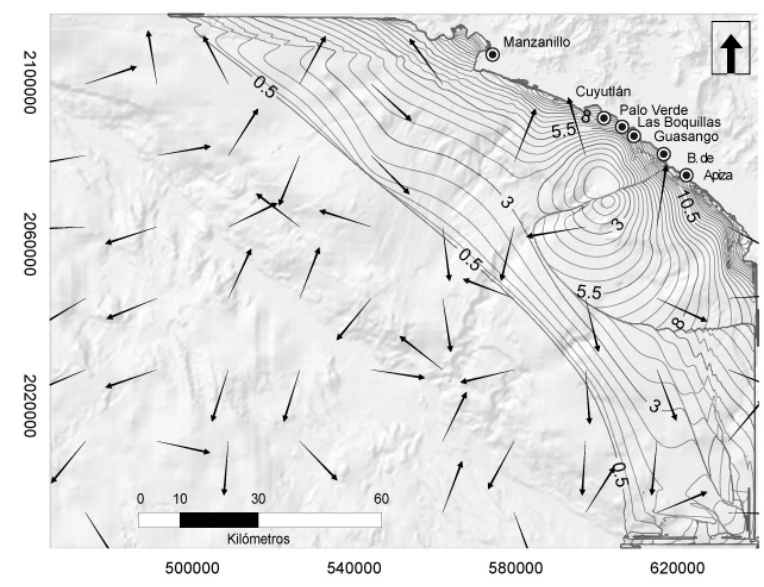

Fuente: Elaboración propia.

co localidades afectadas: Manzanillo, El Real, Salinas del Guasango, Tenancingo y Cuyutlán, solo para el último se registró el valor de altura máxima del tsunami, con un valor de $10 \mathrm{~m}$. Reportes posteriores plantean que el valor pudo ser mayor de $10 \mathrm{~m}$ (Salazar, 1985, 1989; Farreras \& Sánchez, 1991) y hasta 12 m (Corona \& Ramírez-Herrera, 2012), valores que coinciden con los del modelo de generación por deslizamiento (Figura $\mathrm{N}^{\circ} 7$ ).

En el caso de la localidad Palo Verde, en donde las referencias históricas indican que fue totalmente destruido, los valores de $8 \mathrm{~m}$ de altura del tsunami resultantes en el MGTD pudieron haber ocasionado dichos efectos; tomando en cuenta que las casas de madera (material de construcción más empleado en la zona en aquel entonces) son potencialmente derribadas cuando la inundación es de por lo menos de 2 m (Hatori, 1964; Koshimura et al., 2009; Reese et al., 2011). Dado que en el MGTS las alturas están por debajo de 1 $\mathrm{m}$ de altura, se asume que el valor del MFGD es capaz de explicar la magnitud de los daños mencionados en las fuentes históricas (Figura $\mathrm{N}^{\circ} 7$ ).

Por otro lado, el trabajo postsísmico efectuado por Cumming (1933), reporta que los daños ocasionados por el tsunami afectaron de forma proporcional a las localidades de Palo Verde y el Guasango, situadas a 3 y 21 km al SE de Cuyutlán, información que se ratifica en datos de prensa, en donde se señala que la localidad del Tecuanillo (en aquel entonces localizado junto a las salinas del Guasango) también sufrió daños (El Universal Gráfico, 1932b). Interpretando los valores de altura máxima del MGTD, de $8 \mathrm{~m}$ en Palo Verde y $6.4 \mathrm{~m}$ en el Guasango, inferimos que las dimensiones a las que alude Cumming (1933) y las notas de prensa, cobran mayor sentido que en el MGTS, ya que en este los valores resultantes estuvieron por debajo de $1 \mathrm{~m}$. Por otro lado, Salazar (1989) señala que en la zona conocida como "Las Boquillas", actual localidad de El Paraíso, a 18 km al SE de Cuyutlán, se unió el mar con el estero. En este punto los valores de altura máxima del MGTD de $8.7 \mathrm{~m}$ reflejan este comportamiento (Figura $\mathrm{N}^{\circ} 7$ ).

Respecto a las zonas de menor afectación, que no cuentan con datos precisos de la altura que alcanzó el tsunami, ni reportes de daños asociados a este, como es el caso de la bahía de Manzanillo, que no sufrió desperfectos (El Universal Gráfico, 1932a), se reporta que parte de los damnificados se 
Figura $\mathrm{N}^{\circ} 7$

Comparativo de alturas máximas del tsunami, datos histórico-etnográficos vs. Datos de la modelación numérica. Símbolos: Los óvalos señalan las zonas en las que se recabaron datos por medio de técnicas histórico-etnográficas, la intersección del dato de la modelación de tsunami generado por deslizamiento y una breve descripción de las afectaciones en cada sitio.

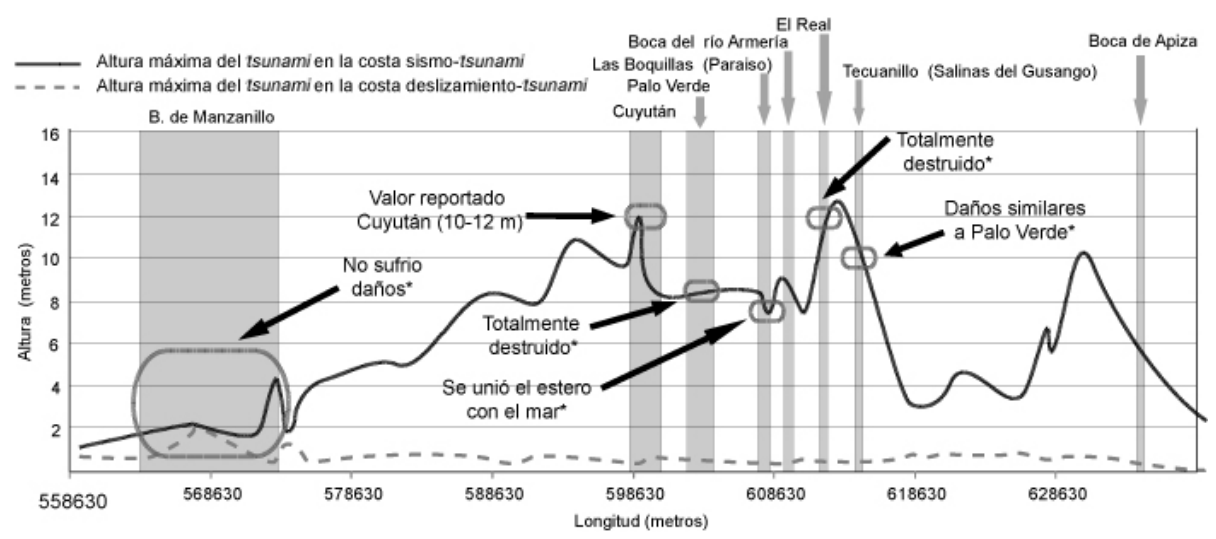

\begin{abstract}
* Observaciones que corresponde con los sitios que registraron el tsunami, pero que carecen del dato de la altura máxima del tsunami.

Fuente: Elaboración propia.
\end{abstract}

refugiaron en Manzanillo (El Universal Gráfico, 1932b), y que la población de Manzanillo participó activamente asistiendo al desastre de Cuyutlán (Excelsior, 1932a). Por tanto, asumimos que los datos de alturas máximas resultantes en el MGTD, de entre 1 y $2.3 \mathrm{~m}$ reflejan el comportamiento del tsunami en esta zona (Figura $N^{\circ} 7$ ). El extremo sur, Boca de Apiza, sin datos históricos, solo ha sido referido para señalar que hasta esa zona pudo haber llegado la perturbación del tsunami. Como se observa en el MGTD (Figuras $N^{\circ} 5$ y 7 ), en esta región extrema de la costa, los valores de altura máxima apenas rebasan valores de altura de $2 \mathrm{~m}$, lo cual no representa amenazas significativas en los poblados localizados en esas zonas.

La longitud de $25 \mathrm{~km}$ de afectación por el tsunami (El Informador, 1932b; Excelsior, 1932a, 1932e; Cumming, 1933; Soloviev \& Go, 1975; Farreras \& Sánchez, 1991; Sánchez y Farreras, 1993; National Geophysical Data Center, 2011; Novosibirsk Tsunami Laboratory, 2011), coincide con los segmentos de costa que concentran los mayores valores, alturas máximas del tsunami en el MGTD, entre las localidades de Cuyutlán y Tecuanillo (Figuras $\mathrm{N}^{\circ} 7$ ).

\section{Tiempos de arribo}

Cuyutlán es la única localidad que cuenta con el dato de tiempo de arribo, reportado como $5 \mathrm{~min}$. Considerando que el MGTS presentó valores de entre 12 y 12.5 min, mientras que el MGTD resultó en valores de 8.5 min, asumimos que al presentar un valor más próximo al observado, puede satisfacer la condición de tiempo de arribo del tsunami (Figura $\mathrm{N}^{\circ} 8$ ).

\section{Directividad}

La dirección de propagación del tren de ondas del tsunami resultante del MGTD se ajusta a las descripciones de Cumming (1933:73), quien señala que: "Por la dirección que tomaron los árboles y postes arrasados frente a la playa de Palo Verde y El Guasango, parece que el centro de propagación del tsunami está situado a unos $10 \mathrm{~km}$ al S-SW de la Boca de Pascuales" (c.a. 3 km al SE de Las Boquillas); por lo tanto, se asume 
Figura $\mathrm{N}^{\circ} 8$

Comparativo de los tiempos de arribo por localidad, modelos vs. Datos históricos-etnográficos.

Símbolos: El óvalo señala el lugar en donde se encontraron datos con métodos histórico-etnográficos.

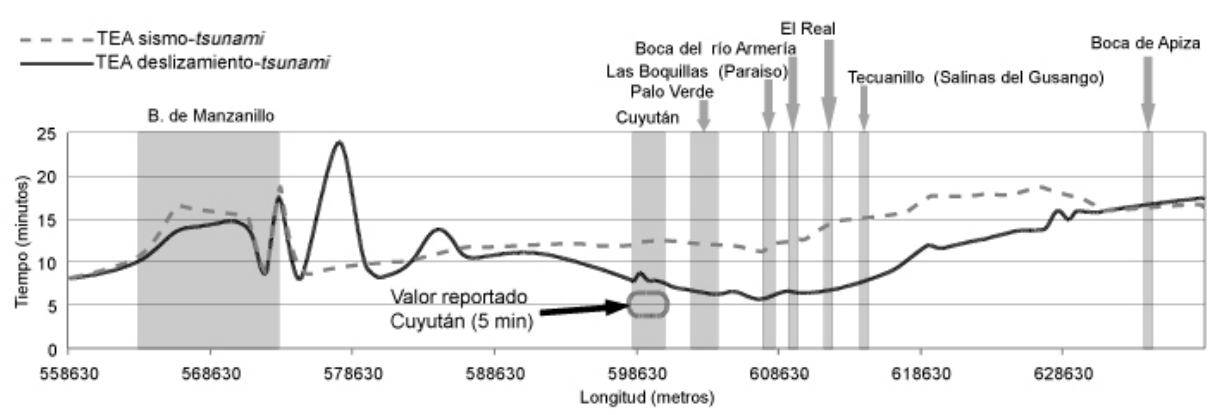

Fuente: Elaboración propia.

que el componente de directividad coincide con el MGTD.

\section{Conclusiones}

La información colectada con técnicas histórico-etnográficas, representados espacialmente con técnicas de localización geográfica, permitió reconstruir y caracterizar el comportamiento del tsunami del 22 de junio de 1932 en las costas de Colima. A partir de esta información, en su mayoría cualitativa, se realizaron dos ejercicios de modelación de tsunami usando las dos hipotéticas fuentes y mecanismos de generación, por sismo y por un deslizamiento submarino. Al comparar los datos histórico-etnográficos con el comportamiento del tsunami 22 de junio, resultante de los modelos aplicados, concluimos que el tsunami se ajusta a un patrón de comportamiento característico de tsunamis generados por deslizamientos submarinos.

Al emplear métodos combinados de distintas disciplinas se logró identificar y definir la causa y los mecanismos de generación, uno de los eventos más destructivos en la zona de estudio. De igual forma se demostró que esta estrategia de investigación permite generar el conocimiento necesario de fenómenos naturales que no cuentan con mediciones instrumentales.
Al esclarecer los mecanismos de generación del tsunami del 22 de junio de 1932, contribuimos a reducir el porcentaje de tsunamis atribuidos a causas desconocidas o poco claras.

\section{Referencias bibliográficas}

BARNIKEL, F. The value of historical documents for hazard zone mapping. Natural Hazards and Earth System Sciences, 2004, N ${ }^{\circ}$ 4, p. 599-613.

BARRIENDOS, M.; COEUR, D.; LANG, M.; LLASAT, M.-C.; NAULET, R.; LEMAITRE, F. \& BARRERA, A. Stationarity analysis of historical flood series in France and Spain (14th20th centuries). Natural Hazards and Earth System Sciences, 2003, Nº 3, p. 583-592.

BIRKMANN, J. \& FERNANDO, N. Measuring revealed and emergent vulnerabilities of coastal communities to tsunami in Sri Lanka. Disasters, 2008, N³2, p. 82-105.

CORONA, N. \& RAMÍREZ-HERRERA, M.T. Revealing the origin of the great tsunami of June 22, 1932 off the Pacific Coast of Mexico. Geology, 2012a, № (Sometido el 16 de Julio de 2012). 
CORONA, N. \& RAMÍREZ-HERRERA, M. T. Mapping and historical reconstruction of the great Mexican 22 June tsunami. Natural Hazards and Earth System Sciences, 2012b, $\mathrm{N}^{\circ} 12$, p. 1337-1352.

CRUZ, G. \& WYSS, M. Large earthquakes, mean sea level, and tsunamis along the Pacific Coast of Mexico and Central America. Bulletin of the Seismological Society of America, $1983, N^{\circ} 73$, p. 553-570.

CUMMING, J. L. Los terremotos de Junio de 1932 en los estados de Colima y Jalisco. Universidad de México, 1933, № 31-32, p. 68-104.

CHÁVEZ, L. Dramático relato de la reciente catástrofe del balneario de Cuyutlán. El Nuevo Mexicano, Santa Fe, Nuevo México, Julio 21, 1932, Sección I, p. 2.

EISSLER, H. K. \& MCNALLY, K. C. Seismicity and Tectonics of the Rivera Plate and Implications For the 1932 Jalisco, Mexico, Earthquake. Journal of Geophysical Research, $1984, N^{\circ} 89$, p. $4520-4530$.

EL INFORMADOR. Han sido formidables los recientes terremotos. El Informador, MéxiCo, Junio 28, 1932a, Sección II, p. 4.

EL INFORMADOR. La catástrofe ocurrida anteayer en Cuyutlán no tiene precedente. EI Informador, México, Junio 24, 1932b, Sección I, p. 1-2.

EL UNIVERSAL GRÁFICO. En Cuyutlán no quedó piedra sobre piedra. El Universal Gráfico, México, Junio 23, 1932a, Sección I, p. 15.

EL UNIVERSAL GRÁFICO. En el risueño balneario no queda ahora sino una media docena de personas. El Universal Gráfico, México, Junio 23, 1932b, Sección I, p. 2.

EL UNIVERSAL GRÁFICO. Las primeras gráficas de la catástrofe de Cuyutlán. EI Universal Gráfico, México, Junio 25, 1932c, Sección I, p. 1.

EXCELSIOR. Cuyutlán arrasado por la invasión del océano: gigantescas olas arrasaron las casas y la gente. Excelsior, México, Junio 23, 1932a, Sección I, p. 1, 4.

EXCELSIOR. Dos veces más Cuyutlán ha sido barrido por las olas. Excelsior, México, Junio 24, 1932b, Sección I, p. 1.

EXCELSIOR. Gigantesco derrumbe submarino a causa de los sismos en Colima. Excelsior, México, Junio 25, 1932c, Sección I, p. 4-5.

EXCELSIOR. Mil doscientos huyen hacia Colima. Excelsior, México, Junio 24, 1932d, Sección II, p. 3.

EXCELSIOR. Una extensa zona marítima afectada por el sismo de ayer. Excelsior, México, Junio 23, 1932e, Sección 1, p. 1.

FARRERAS, S. F. \& SÁNCHEZ, A. J. The tsunami threat on the Mexican west coast: A historical analysis and recommendations for hazard mitigation. Natural Hazards, 1991, № 4, p. 301-316.

FLIRI, F. Naturchronik von Tirol. Innsbruck: Universitätsverlag Wagner, 1998.

FUKAO, Y. Tsunami earthquakes and subduction processes near deepsea trenches. Journal of Geophysical Research, 1979, № 84, p. 2303-2314.

GIANNECCHINI, R. \& D'AMATO AVAN$\mathrm{ZI}, \mathrm{G}$. Historical research as a tool in estimating hydrogeological hazard in a typical small alpine-like area: The example of the Versilia River basin (Apuan Alps, Italy). Physics and Chemistry of the Earth, Parts $A / B / C, 2012, N^{\circ}$ 49, p. 32-43.

GUHA-SAPIR, D.; VOS, F.; BELOW, R. \& PONSERRE, S. Annual Disaster Statistical Review 2011: The Numbers and Trends. Brussels: CRED, 2012.

HATORI, T. A Study of the Damage to Houses due to a Tsunami. Bulletin of the Earthquake Reasearch Institute, 1964, № 42, p. 181-191.

JANKAEW, K.; ATWATER, B. F.; SAWAI, Y.; CHOOWONG, M.; CHAROENTITIRAT, T.; MARTIN, M. E. \& PRENDERGAST, A. Me- 
dieval forewarning of the 2004 Indian Ocean tsunami in Thailand. Nature, 2008, $\mathrm{N}^{\circ} 455, \mathrm{p}$. 1228-1231.

KOSHIMURA, S.; NAMEGAYA, Y. \& YANAGISAWA, H. Tsunami Fragility: A New Measure to Identify Tsunami Damage. Journal of Disaster Research, 2009, № 4, p. 479-480.

KULIKOV, E. A.; RABINOVICH, A. B. \& THOMSON, R. E. Estimation of Tsunami Risk for the Coasts of Peru and Northern Chile. Natural Hazards, 2005, N³5, p. 185-209.

LØVHOLT, F.; GLIMSDAL, S.; HARBITZ, C. B.; ZAMORA, N.; NADIM, F.; PEDUZZI, P.; DAO, H. \& SMEBYE, H. Tsunami hazard and exposure on the global scale. Earth-Science Reviews, 2012, №110, p. 58-73.

LUHR, J. F.; NELSON, S. A.; ALLAN, J. F. \& CARMICHAEL, I. S. E. Active rifting in southwestern Mexico: Manifestations of an incipient eastward spreading-ridge jump. Geology, 1985, №13, p. 54-57.

LLASAT, M.-C.; BARRIENDOS, M.; BARRERA, A. \& RIGO, T. Floods in Catalonia (NE Spain) since the 14th century. Climatological and meteorological aspects from historical documentary sources and old instrumental records. Journal of Hydrology, 2005, $N^{\circ} 313$, p. 32-47.

MATUTE, J. Croquis de los límites del Departamento de Colima / Formado a vista por los ingenieros D. Juan B. Matute y D. Miguel M. Orozco, Escala de 10 leguas. México: MAPAMEX, 1865.

NATIONAL GEOPHYSICAL DATA CENTER. Global Historical Tsunami Database. 2011. Disponible en Internet: http://www. ngdc.noaa.gov/hazard/tsu_db.shtml

NIXON, G. T. The relationship between Quaternary volcanism in central Mexico and the seismicity and structure of subducted ocean lithosphere. Geological Society of America Bulletin, 1982, N 93, p. 514-523.

NOVOSIBIRSK TSUNAMI LABORATORY. Historical Tsunami Database for the World Ocean, 2011. Disponible en: http://tsun.sscc. ru/nh/tsun_descr.html
OKAL, E. A. \& BORRERO, J. C. The 'tsunami earthquake' of 1932 June 22 in Manzanillo, Mexico: seismological study and tsunami simulations. Geophysical Journal International, 2011, No 187 , p. 1443-1459.

ORDÓÑEZ, E. Seismic activity in Mexico during June, 1932. Bulletin of the Seismological Society of America, 1933, No 23, p. 80-82.

REESE, S.; BRADLEY, B. A.; BIND, J.; SMART, G.; POWER, W. \& STURMAN, J. Empirical building fragilities from observed damage in the 2009 South Pacific tsunami. Earth-Science Reviews, 2011, № 107, p. 156173.

RYAN, W. B. F.; CARBOTTE, S. M.; COPLAN, J. O.; O'HARA, S.; MELKONIAN, A.; ARKO, R.; WEISSEL, R. A.; FERRINI, V.; GOODWILLIE, A.; NITSCHE, F.; BONCZKOWSKI, J. \& ZEMSKY, R. GIobal Multi-Resolution Topography synthesis. Geochemistry Geophysics Geosystems, 2009, $N^{\circ} 10$, p. Q03014.

SALAZAR, J. Una Montaña de Agua. Diario de Colima, Colima, 1985, Sección Agora: Suplemento cultural del Diario de Colima, p. 2-3.

SALAZAR, J. El maremoto de Cuyutlán, 1932. Colima, México: Sociedad Colimense de Estudios Históricos, 1989.

SÁNCHEZ, A. J. y FARRERAS, S. F. Catálogo de Tsunamis (Maremotos) en la Costa Occidental de México. Boulder: National Geophysical Data Center, 1993.

SERVICIO SISMOLÓGICO NACIONAL. Catálogo de sismos. Disponible en Internet: http://www.ssn.unam.mx/

SINGH, S. K.; ASTIZ, L. \& HAVSKOV, J. Seismic gaps and recurrence periods of large earthquakes along the Mexican subduction zone: A reexamination. Bulletin of the Seismological Society of America, 1981, N 71, p. 827-843.

SINGH, S. K.; PACHECO, J. F. \& SHAPIRO, N. The earthquake of 16 November, $1925(\mathrm{Ms}=7.0)$ and the reported tsunami in 
Zihuatanejo, Mexico. Geofísica Internacional, 1998, № 37, p. Short Note.

SINGH, S. K.; PONCE, L. \& NISHENKO, S. P. The great Jalisco, Mexico, earthquakes of 1932: Subduction of the Rivera plate. Bulletin of the Seismological Society of America, 1985, N 75 , p. 1301-1313.

SOLOVIEV, S. L. \& GO, C. N. Catalogue of tsunamis on the eastern shore of the Pacific Ocean. Moscow: Nauka Publishing House, 1975.

STEIN, S. Limitations of a Young Science. Seismological Research Letters, 2006, №77, p. 351-353.

USHA, T.; MURTHY, M. V. R.; REDDY, N. T. \& MISHRA, P. Tsunami vulnerability assessment in urban areas using numerical model and GIS. Natural Hazards, 2012, N ${ }^{\circ}$ 60, p. 135-147.

VALDIVIA-ORNELAS, L.; CASTILLO-AJA, M. D. R. \& ESTRADA-TREJO, M. Elements for an historical review of the 3 June, 1932 tsunami on the coast of Jalisco, Mexico. American Geophysical Union, 2011.

WANG, P. \& ZHANG, D. An introduction to some historical governmental weather records of China. Bulletin of the American Meteorological Society, 1988, № 69, p. 753-758.

WYSS, M.; HABERMANN, R. \& GRIESSER, J.-C. Seismic Quiescence and Asperities in the Tonga-Kermadec Arc. Journal of Geophysical Research, 1984, No 89, p. 92939304. 